\title{
COMPASSIO ALS HELDENTUGEND AM BEISPIEL DES „WILLEHALM“-FRAGMENTS: ZUR DARSTELLBARKEIT VON GEFÜHLEN IN DER EPENILLUSTRATION
}

\author{
VON LIESELOTTE E. SAURMA-JELTSCH
}

\section{Affekte in Bild und Text}

Zornbebend steht Willehalm vor seinen Verwandten und durchbricht sämtliche Formen gesellschaftichen Umgangs, indem er vor versammeltem Hofe seiner Schwester aus Wut über ihre mangelnde Familiensolidarität ${ }^{1}$ die Krone vom Kopf reißt ${ }^{2}$ und zu Boden wirft. Do begreif der zornbaere gast / bi den zöpfen die künegin ${ }^{3}$ und bedroht gar ihr Leib und Leben. Wut und Zorn verleiten den Helden zu einem schändlichen Verhalten, verliert er doch nicht allein die geforderte Kontenance ${ }^{4}$, sondern verstößt sogar gegen das Gebot des höfischen Umgangs mit Frauen, dem er als Gast in besonderem Maße unterstellt gewesen wäre. An solch großen Gefuihlen, die zu Normbrüchen drängen, fehlt es in den Epen des Hochmittelalters wahrlich nicht: Wegen ihrer unmäßigen Minne dem Wahn verfallen, versucht sich Dido an Eneas zu rächen ${ }^{5}$, und Sieglinde dämmert sinnelos vor Trauer nach der Ermordung ihres Gatten dem Tod entgegen ${ }^{6}$. Wut, Freud und Leid, Liebe und Schmerz, Neid und Mißgunst treiben in diesen Dichtungen die Akteure in Extremsituationen und werden - obwohl in solchem Unmaß gesellschaftlich nicht erlaubt ${ }^{7}$ - aus dem jeweiligen Zusammenhang dennoch verständlich gemacht.

Während also die Literatur der Gemüts- und insbesondere der Affektlage ihrer Helden ein breites Interesse widmet, bleiben die in den entsprechenden Illustrationen dargestellten Personen unberührt von jeglichen Emotionen. Bezeichnend für das Ausblenden dieses The-

1) E. SCHXUfEIE, Normabweichendes Rollenverhalten. Die kämpfende Frau in der deutschen Literatur des 12. und 13. Jahurhunderts, phil. Diss. (Göppinger Arbeiten zur Germanistik, hrsg. von U. Mouler u. a., 272), Göppingen 1979, S. 48 f. - Zu weiteren Beispielen von affektbedingten Rollenübertretungen vgl. Willehalm, VV. 153, 15 ff. Zitiert wird im folgenden nach: WOLfRAM vON EsCHENBACH, Willehalm, hrsg. v. W. SCHRODER, völlig neubearbeitete Übersetzung von D. KarTschoKE, Berlin/New York 1989.

2) Willehalm (zit. Anm. 1), VV. 145, 4.

3) Ebenda, V. 147, $18 \mathrm{f}$.

4) Damit hat Willehalm nicht nur gegen das Gebot der temperantia verstoßen, also gegen den Begriff des höfischen Verhaltens, sondern hat auch die Pflicht des Gastes außer acht gelassen; vgl. dazu J. BumKE, Höfische Kultur. Literatur und Gesellschaft im hohen Mittelalter, München 1986, bes. Bd. 2, S. $416 \mathrm{ff}$; dort weitere Literatur.

5) HeINRICH von Veldeke, Eneas, nach dem Text von L. ETtmoller überabeitet von D. Kartschoke, Stuttgart 1986, VV. $761 \mathrm{ff}$.

6) Das Nibelungenlied, hrsg. von H. BraCKERT, Frankfurt a. M. 1970, VV. 1070.

7) Zum Gebot der Mäßigung vil. M. BARASH, Gestures of Despair in Medieval and Early Renaissance Art, New York 1976, S. $34 \mathrm{ff}$. 
mas ist gerade die bildnerische Gestaltung Willehalms in seinem eben erwähnten Zornesausbruch. In der Version des frühen 14. Jahrhunderts, in der Wiener Handschrift (Abb. 1), ist nichts von des Helden Empörung zu erkennen. Im Gegenteil ist hier das skandalöse Ereignis - das Herabreißen der Krone vor versammelter Hofgesellschaft - sogar mit einer positiven Konnotation versehen, identifiziert man doch den Akt zunächst als Krönung8. Keine Emotionen stören die hier gewählte Szene eines zeremonialisierten Gastmahls. Einzig am unbotmäßigen Griff zum Schwert erkennt der Betrachter - nicht jedoch die Mitakteure - die aggressive Haltung Willehalms9.

Die vermutete Scheu vor der Darstellung der maßlosen, die Formen verletzenden Gefühlsäußerung wird im Beispiel des Wiener „Willehalm" noch insofern bestätigt, als auch die Reaktionen der übrigen Akteure ausgeblendet werden. In gelassener Unberührtheit kommen alle Teilnehmer an dieser Szene ihrer Pflicht zur zuchtvollen „höveschheit ${ }^{\alpha}$ nach. Lediglich der Normenverstoß - hier der unerlaubte Griff zum Schwert - wird in verschlüsselter Form angedeutet. Eine betonte Zurückhaltung gegenüber der bildnerischen Darstellung von Affekten hängt zweifellos auch mit der spezifischen Entwicklung der Buchillustration als Medium sowie der besonderen Beziehung der Bilder zu den Texten zusammen, auf die hier nicht ausführlicher eingegangen werden $k^{2} n^{10}$. Es sei nur darauf hingewiesen, daß das erstmalige Darstellen von Willehalms Zornesausbruch in der Wolfenbütteler Handschrift ${ }^{11}$ aus dem letzten Viertel des 14. Jahthunderts im Rahmen dieser Entwicklung zu sehen ist, wird doch dann im 15. Jahrhundert etwa auch Dietrichs rauchende Wut ${ }^{12}$ oder Didos Liebeswahn gestaltet ${ }^{13}$. In den frühen Epenillustrationen des 13. und auch noch des 14. Jahrhunderts hingegen werden Emotionen und Affekte entweder - wie eben beobachtet - in Gesten verschlüsselt oder gleichsam an Gefühlsträger ausgelagert. Eine entsprechende Funktion übernehmen beispielsweise die Schriftrollen in der Berliner ${ }_{\text {Eneide }}{ }$, manifestiert sich dort doch auf eindrucksvolle Weise etwa Didos Haß und Wut in dem steil aus ihrem Mund herausschießenden Schriftband, mit dem sie dem abfahrenden Eneas nachbrüllt ${ }^{14}$.

8) Der Griff zur Krone wird hier vom Bildtypus und der „intakten“ Umgebung her zunächst als ein Aufsetzen interpretiert; einzig der Titulus bie rouft der markeis di chuniginn sein schwester sowie das Ziehen des Schwertes sind Hinweise auf eine andere Deutung des Bildgeschehens.

9) Das Tragen oder gar Zücken von Waffen vor einer Gesellschaft wird als Affront empfunden, dazu etwa Nibelungenlied (zit. Anm. 6), V. 418, 2: die Prünhilde recken. die truogen swert enhant, / fünf hundert oder mère, daz was den gesten leit.

10) Zur unterschiedlichen Behandlung in Literatur und Kunst vgl. BArasH, Gestures (zit. Anm. 7), S. 90 ff.

ii) Wolfenbüttel, Herzog August Bibliothek: Cod. guelf. 30. 12 Aug. fol., fol. 112; Abb. s. R. M. SCHMIDT, Die Handschriftenillustrationen des "Willehalm" Wolframs von Eschenbach, Wiesbaden 1985, Abb. 69. Thematisiert ist hier der Angriff auf der Schwester Leben, wobei die Ikonographie der Malchusszenen paraphrasiert wird.

12) Heidelberg, Universitätsbibliothek: Cpg. 324, fol. 32 bzw. 43: Die Zornesäußerung wird wörtlich ins Bild übersetzt als Feueratem, der aus Dietrichs Mund dringt.

13) In der Berliner "Eneide“ aus dem 13. Jahrhundert, Berlin, Staatsbibliothek, Preußischer Kulturbesitz: Ms. Germ. fol. 282, ist in der Szene der Abfahrt des Eneas (fol. XVII) Dido im Zustand äußerster Verzweiflung gezeigt; ihr Außersichsein allerdings wird erst beim Selbstmord (fol. XVIIv) artikuliert; Abb. s. A. BOECKnER,

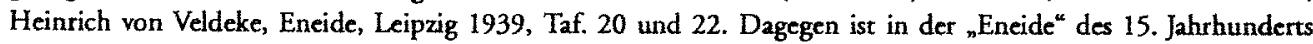
(Heidelberg, Universitätsbibliothek: Cpg. 403, fol. 32v) Didos Liebeswahn in einem eigenen Bild gestaltet, und ihrem verzweiflungsvollen Selbstmord werden gar zwei Bilder gewidmet; vgl. dazu Heinrich von Veldeke, Eneasroman, Farbmikrofiche (Codices illuminati medii aevi, 2), München 1987.

14) Abb. s. BOECXIER, ebenda, Taf. 20; zur Bedeutung der Schriftrollen im Erzählzusammenhang vgl. M. CA MLLE, Seeing and Reading. Some Visual Implications of Medieval Literacy and Illiteracy, in: Art History, 8, 1985, 


\section{Das Gefuibl als sittliches Vorbild am Beispiel der "Großen Bilderhandschriff“}

Vor dem Hintergrund einer solch sparsamen Schilderung individueller Gemütslagen müssen Darstellungen, wie sie in der sogenannten "Großen Bilderhandschrift ${ }^{4}$ des W Willehalm ${ }^{4}$ vorkommen, eine besondere Bedeutung besitzen. In einem einzigen Blatt wird hier (Abb. 3, 4) eine ganze Palette der unterschiedlichen emotionalen Zustände gezeigt, in denen sich der Held Willehalm befindet. Diesen Illustrationen soll der folgende Beitrag gewidmet sein. Dabei gilt es zu analysieren, welche Gefühle darstellbar sind und in welchem Zusammenhang die entsprechenden Äußerungen zulässig werden. Als Arbeitshypothese möchte ich behaupten, daß solche Regungen einzig über die Konnotation der christlichen Ethik zu verbildlichen sind und daß infolgedessen das Epos durch die Bilder eine Rechristianisierung erfährt, ja sogar zu einem Lehrstück für christliche Moral wird.

Bevor wir versuchen, diese Behauptung anhand der Bildanalysen zu belegen, soll zunächst die Besonderheit der sogenannten "Großen Bilderhandschrift" kurz dargestellt werden.

\section{Das Illustrationsschema der „Großen Bilderhandschrift"}

Lediglich elf Fragmente einer ehemals sehr prächtigen Version von Wolframs "Willehalm ${ }^{\text {“ }}$ aus der Zeit um 1270/80 sind uns erhalten geblieben. Diese in der Staatsbibliothek in München ${ }^{15}$ bzw. in Nürnberg im Germanischen Nationalmuseum ${ }^{16}$ aufbewahrten Bruchstücke scheinen den Text beinahe Wort für Wort zu illustrieren ${ }^{17}$. Bereits Karl von Amira hat deshalb die Fragmente als Teile einer "Großen Bilderhandschrift" bezeichnet ${ }^{18}$, deren vermuteter Umfang sie nicht allein unter den „Willehalm"-Codices zum bilderreichsten Exemplar ${ }^{19}$ werden läßt, sondern ihr sogar innerhalb der illustrierten Handschriften dieser Zeit eine Sonderstellung einräumt ${ }^{20}$. Erst wenn man bedenkt, daß damalige Bibeln meist

S. 26-49, zur „Eneide“ vor allem S. 38 f.; N. Henkel, Bildrexte. Die Spruchbänder in der Berliner Handschrift von Heinrichs von Veldeke Eneasroman, in: Poesis et pictura. Festschrift für Dieter Wuttke, hrsg. von S. Fossel und J. KNAPE, Baden-Baden 1989, S. 1-47, bes. S. 19 f.

15) München, Bayerische Staatsbibliothek: Cgm 19/III; s. die vollständige Wiedergabe in: Wolfram von Eschenbach, Willehalm. Die Bruchstuicke der "Großen Bilderhandschrift", Faksimile-Edition hrsg. von U. MONTAG, Stuttgart 1985; zur Literatur vgl. ScHMiDr, Willehalm (zit. Anm. 11), S. 151; vgl. auch Katalog: Meister der Zeichnung. Ausstellung Germanisches Nationalmuseum, Nürnberg 1992, Kat. Nr. 1, S. 14-17.

16) Nürnberg, Germanisches Nationalmuseum: Kapsel 1607, Inv. Nr. Hz 1104 bzw. Inv. Nr. Hz 1105; zur Literatur vgl. Anm. 15.

17) Der Codex dürfte 224 Blätter mit ungefähr 1300 Bildern umfaßt haben; vgl. dazu W. SCHRODER, Text und Bild in der "Großen Bilderhandschrift" von Wolframs "Willehalm", in: Zeitschrift für deutsches Altertum und deutsche Literatur, 116, 1987, S. 239-268, bes. S. 239.

18) K. v. AMIRA, Die große Bilderhandschrift von Wolframs "Willehalm", in: Sizzungsberichte der philosophisch-philologischen und der historischen Klasse der Kgl. Bayerischen Akademie der Wissenschaften zu München, 1903, München 1904, S. 213-240.

19) Wien, Osterreichische Nationalbibliothek: Cod. 2670, umfaBt 117 Bilder; Kassel, Murhardsche und Landesbibliothek: $2^{*}$ Ms. poet. et roman. 1, 62 zum Teil unfertige Bilder; Wolfenbüttel, Herzog August Bibliothek, Cod. guelf. 30.12. Aug. fol., 86 Bilder.

20) Soweit ich sehe, wurde bisher nicht daran gedacht, daß auch eine Teilillustration geplant worden sein könnte. 
nur wenige Miniaturen trugen ${ }^{21}$ und insbesondere die volkssprachlichen Epen nur zögerlich als illustrationswürdig erachtet wurden ${ }^{22}$, ist die Bedeutung dieser Bilderdichte gebührend zu würdigen. Eine vergleichbare Verbildlichung kennen allenfalls Heiligenlegenden ${ }^{23}$, typologische Texte wie etwa die „Bible moralisée“ ${ }^{24}$ sowie didaktisch-theoretische Arbeiten in der Art des "Hortus Deliciarum" der Herrad von Landsberg 25 .

Nicht allein mit ihrer Illustrationszahl, sondern auch mit deren spezifischer Anordnung und Erzählweise entspricht die "Große Bilderhandschrift" nicht den Schemata üblicher Handschriftenausstattungen. Ihre Bilder sind in einer eigenen Kolumne neben die Schrift gesetzt. Die jeweils mit dem Text zu verbindende, der Illustration zugeordnete Initiale schafft überdies eine intensive Verknüpfung zwischen den beiden Medien. Als eine ihr entsprechende Gestaltung wurde seit Karl von Amira diejenige der ${ }_{n}$ Sachsenspiegel ${ }^{{ } 26}$ verstanden. Mit diesen Rechtshandschriften stimmt denn tatsächlich nicht allein das BildText-Verhältnis überein, sondern auch die Textverweise in den Bildern kommen hier wie dort vor ${ }^{27}$. Ebenso vergleichbar ist das Erzählen in Registern, die auffällige Betonung der Gebärdensprache sowie die Verbildlichung abstrakter Inhalte, ein Darstellungsmodus, auf den wir gleich ausfiuhrlicher eingehen müssen. Da nun allerdings vor dem 14. Jahrhundert keine Exemplare dieser Rechtsbücher erhalten sind, wird auf ein Urexemplar geschlossen ${ }^{28}$,

21) Meist konzentriert sich die Ausstattung auf figürliche Initialen zu den Buchanfängen; selbst eine der reichsten Handschriften, Stuttgart, Württembergische Landesbibliothek: Bibl. fol. 60, weist immer noch einen relativ geringen Bilderanteil auf; ebenso trifft dies auf die wenigen Beispiele mit ganzseitigen Bildern etwa in der Art der Riesenbibeln zu, vgl. dazu W. CAHN, Die Bibel in der Romanik, München 1982, S. $154 \mathrm{ff}$.

22) Berlin, Staatsbibliothek, Preußischer Kulturbesitz: Ms. germ. fol. 282; die „Eneide“ verfügt trotz des Bilderreichtums lediglich über etwas mehr als 130 Bildszenen; vgl. dazu BOECKIER, Eneide (xit. Anm. 13). Vom Münchner „Tristan“, Bayerische Staatsbibliothek: Cgm 51, sind auf 30 Seiten 84 Bilder bzw. 120 Szenen erhalten. Auch wenn man hier mit einem Verlust von weiteren 20 Bildseiten rechnen dürfte, würde doch auch diese Handschrift niemals an die Dimensionen der "Großen Bilderhandschrif" herankommen. Vgl. dazu P. GiCHTEL, Die Bilder der Münchener Tristan-Handschrift, in: Gottfried von Straßburg „Tristan und Isolde“. Mit der Fortsetzung Ulrichs von Türheim. Faksimile-Ausgabe des Cgm 51 der Bayerischen Staatsbibliothek München, Textband mit Beiträgen von U. MONTAG und P. GICHTEL, Stuttgart 1979, bes. S. $84 \mathrm{ff}$.

23) So etwa im Lektionar des Desiderius von Montecassino; vgl. dazu B. BrenK, Das Lektionar des Desiderius von Montecassino. Wissenschaftlicher Ergänzungsband zur Faksimileausgabe des Codex Benedictus Vat. Lat. 1202, Zürich 1987.

24) Vgl. dazu R. HaussherR, Bible moralisee, Codex Vindobonensis 2554, Graz - Paris 1973.

25) A. Straub - G. Keller, Herrad of Landsberg, "Hortus Deliciarum“, Neudruck der Ausgabe Straßburg 1899, New York 1977.

26) v. AMIRA, Bilderhandschrift (zit. Anm. 18), S. 239, und die ihm nachfolgende Literatur sieht im Gegenteil in der "Willehalm ${ }^{\prime}$-Ausstattung den Prototyp, was SCHRODER vor allem wegen der einem Epos wesensfremden lllustrierung ablehnt; dazu SCHRODER, Text und Bild (zit. Anm. 17), S. 267 f; vgl. dazu auch M. CurschmanN, Pictura laicorum litteratura? Uberlegungen zum Verhältnis von Bild und volkssprachlicher Schriftlichkeit im Hoch- und Spätmittelalter bis zum Codex Manesse, in: Pragmatische Schriftlichkeit im Mittelalter, hrsg. von H. Keller u. a. (Münstersche Mittelalter-Schriften, 65), 1992, S. 211-229, bes. S. 220, Anm. 32, dort weitere Literatur; vgl. auch N. H. Orr, Der Körper als Hülle des Abstrakten. Zum Wandel der Rechtsgebärde im Spätmittelalter, in: Gepeinigt, begehrt, vergessen, hrsg. von K. SCHREINER und N. SCHNITZIFr, München 1992, S. 223-241, bes. S. 223. - Zur älteren Literatur über die Beziehung zwischen Sachsenspiegel und „GroBer Bilderhandschrift" vgl. SCHRODER, ebenda, S. $242 \mathrm{f}$.

27) Abb. s. Der "Sachsenspiegel" in Bildern, hrsg. von W. KosCHOrRecK, Frankfurt 1976, bes. S. 32.

28) SCHRODER, Text und Bild (zit. Anm. 17), Anm. 6 und 8. 
in welchem dieser Typus der Illustrierung entwickelt und dann auf die Epenhandschrift als Fremdform übertragen wurde ${ }^{29}$.

Allerdings scheint zumindest eine weitere, bisher nicht beachtete Kategorie von Handschriften ebenfalls gewisse Verwandtschaften zu dieser Erzählstruktur zu besitzen. Theologische Werke, vor allem der didaktischen Literatur, lassen eine Reihe von Übereinstimmungen erkennen. In den beiden erhaltenen Handschriften des sogenannten Gebetbuchs der Hildegard von Bingen ${ }^{30}$ findet sich beispielsweise zur Bergpredigt und zu den Seligpreisungen $^{31}$ eine Wort-für-Wort-Illustration, in welcher nicht nur die Bilder in einer der ${ }_{n}$ Großen Bilderhandschrift ${ }^{a}$ entsprechenden Kolumnenreihung aufgeführt sind, sondern überdies die auf die Gesten konzentrierte, lapidare Erzählweise eine innere Verwandtschaft aufweist ${ }^{32}$. Gerade diese Bemühungen um die Verbildlichung von abstrakten Inhalten und um eine besondere Klarheit der Erzählung mit Hilfe überdeutlicher Gesten sind Elemente, die der "Großen Bilderhandschrift" nach Meinung der bisherigen Autoren nicht zum Ruhm gereichen ${ }^{33}$, die aber, wie wir noch ausführen werden, gerade in theologisch-didaktischen Illustrationen in der Art der Hildegard-Gebetbücher durchaus ihren Platz haben.

Ein möglicher Bezug zu solchen Werken lockert die bisher angenommene enge Bindung zu den Sachsenspiegeln ${ }^{34}$ und schafft Raum für neue Interpretationsversuche der Bilder und deren Funktionen. Denkbar wäre nun auch die Übertragung und Modifizierung eines Illustrationsmodells auf zwei unabhängige Gattungen und deren gleichzeitige Variierung. Dies würde bedeuten, daß der Typus - obwohl er auch in den Sachsenspiegeln vertreten ist - im Epos keinenswegs mit denselben Funktionen verbunden sein muß. Ja, die Verbindung zu den Hildegard-Gebetbüchern läßt sogar die Frage aufkommen, ob hier nicht mit einer Herleitung von zwei verschiedenen Quellen und entsprechend unterschiedlichen inhaltlichen Implikationen zu rechnen sei?

Um so drängender allerdings wird damit die Frage nach einem Sinn dieses „dichtungsfremden Experiments ${ }^{{ }^{35}}$. Denn darüber besteht kein Zweifel, daß eine solch paratakrische Aufsplitterung des Textes in den Bildern sowie deren Versuche, Sprache und Denken gleicherweise wiederzugeben, dem Fluß des Epos zuwiderlaufen. Gehen wir jedoch davon aus, diese Entfremdung des Textes mit Hilfe der Bilder liege geradezu in der Intention der Hersteller, was ja eigentlich bereits die besondere Dichte der Bebilderung nahelegt, so können nur die Illustrationen selbst uns Aufschluß über ihre Funktion geben.

29) Zur Literatur vgl. Anm. 26.

30) München, Bayerische Staatsbibliothek: Clm 935. Faksimile-Ausgabe hrsg. von E. KLEMM, Wiesbaden 1982; Wien, Osterreichische Nationalbibliothek: Cod. 2739*; dazu E. KıEMM, Das sogenannte Gebetbuch der Hildegard von Bingen, in: Jahrbuch der kunsthistorischen Sammlungen in Wien, 74, NF. 38, 1978, S. 29-78.

31) Matthäus 3, 1-11.

32) J.-CL. SChMrT, La raison des gestes dans l'occident médiéval, Paris 1990, S. 154-172 (dt.: Die Logik der Gesten im europäischen Mittelalter, Stuttgart 1992; im folgenden wird nach der französischen Ausgabe zitiert).

33) Vgl. dazu Schroder, Text und Bild (zit. Anm. 17), S. 242 und S. 267 f.; vgl. auch Curschmann, Pictura (zit. Anm. 26), S. 220.

34) Der paläographische Befund legt eine Datierung der ${ }_{n}$ Großen Bilderhandschrific um 1270 nahe; vgl. dazu MONTAG, Willehalm (zit. Anm. 15), S. 48; vgl. auch Katalog: Meister (zit. Anm. 15), S. 17, datiert um 1260/70. Die Illustrationen würden $m$. E. sogar noch eine etwas spätere Datierung erlauben. Dies würde bedeuten, daß mit einer ungefähren Gleichzeitigkeit der ersten "Sachsenspiegel" und der "Großen Bilderhandschrif" ${ }^{*}$ zu rechnen wäre.

35) SCHroder, Text und Bild (zit. Anm. 17), S. 268. 
Grundlage hierzu sollen - wie oben schon erwähnt - die beiden Blätter sein, in denen Willehalms Zorn auf seine Schwester bewältigt wird (Abb. 3, 4). In diesen Darstellungen wird unser Hauptaugenmerk auf die ungewöhnliche Schilderung von gefühlsgeladenen Situationen gerichtet sein. Eine gewisse Konkretisierung der hierbei analysierten Bildaussagen wird uns ein Blick auf einen Ausschnitt aus dem Religionsgespräch zwischen Giburg und ihrem Vater Teramer (Abb. 5,6) erlauben.

\section{Die Formen der Caritas}

In der oberen Szene des Münchner Fragments (Abb. 3) erhält der eben von Alyze besänftigte ${ }^{36}$ Willehalm von seiner Mutter Irminschart das Angebot der Waffenhilfe für den Kampf gegen die seine Gemahlin Giburg vor Orange bedrängenden Heiden ${ }^{37}$. In voller Rüstung, mit gegürtetem Schwert, zeigt Willehalm auf den Helm und den Schild, der den ihm zugehörigen Stern trägt, und nimmt per Handschlag jenes Angebot der Mutter an, das der Sitte entspricht, nämlich die Finanzierung der Kriegskosten. Diese Hilfe ist mit den gelben und weißen Scheiben angedeutet, auf die Irminschart hinweist. In der Szene sind somit zwei Vorgänge festgehalten: Willehalm schließt mit seiner Murter den Unterstützungsvertrag $^{38}$ und, indem er damit auch das durch das Fehlverhalten seiner Schwester gestörte verwandtschaftliche Beziehungsnetz wiederherstell ${ }^{39}$, rückt er zugleich mit aller Entschiedenheit die aus den Fugen geratene gesellschaftliche Ordnung wieder zurecht: Triuwe... und pris (V. 161, 12) geben ihm voleclichen trost (V. 161, 13); nicht die mit dem Schwert losschlagende Mutter (V. 161, 10), sondern verwandtschaftliche Treue und finanzielle Hilfe liefern ihm Trost ${ }^{40}$. Im Handschlag wird also nicht allein der Unterstützungsvertrag besiegelt, sondern damit wird auch der Verstoß gegen die Ordnung beigelegt, den die Schwester begangen hat: das Vorziehen der Eigenliebe gegenüber der Verwandtenliebe ${ }^{41}$. Indem sie nämlich ihr egoistisches Interesse (V. 147,6) voranstellte, hatte sie sich in gravierender Weise gegen das Gebot der christlichen Liebe vergangen, die sich gerade dadurch definiert, daß man sich selbst und den Nächsten Gottes wegen liebt ${ }^{42}$. Die uneigennüizige Verwandtenliebe - eine Form der Caritas - wird hier von Willehalm und seiner Mutter per Handschlag bestätigt. In diesem Akt dürften allerdings zwei verschiedene Bedeutungsschichten angesprochen sein. Die Verwandtenliebe nämlich ist - gemäß Wilhelm von St.-Thierry,

36) Willehalm (zit. Anm. 1), VV. $154 \mathrm{ff}$.

37) Willehalm, ebenda, VV. 160, 20-161, 30.

38) Zur Bedeutung der Handreichung als „Gebärde des vertraglichen Gelöbnisses“ vgl. KoschORRECK, Sachsenspiegel (zit. Anm. 27), S. 78; vgl. auch ScHmmt, Gestes (zit. Anm. 32), S. 329; zum mehrschichtigen Sinn dieser Gebärde als Kommunikation und als juridischer Vorgang vgl. OT, Körper (zit. Anm. 26), S. 225.

39) Zum Stellenwert von Verwandtschaft im „Willehalm" vgl. CHR. KrenING, Reflexion-Narration: Wege zum „Willehalm" Wolframs von Eschenbach, phil. Diss., Tubingen 1991, S. $194 \mathrm{f}$.

10) Zur ambivalenten Darstellungsweise der kämpfenden Frau vgl. SchäufelE (zit. Anm. 1), S. 90 f.

41) Eine entsprechende Interpretation findet sich auch in Willehalm (zit. Anm. 1), VV. $158 \mathrm{ff}$.

42) Caritas enim Deum facit diligi propter se et proximum Dewm (. . .) Bonaventura. Commentaria in I-IV libros Sententiarum III S d 27 a 294; zit. nach M. SCHLosser, Cognitio et amor. Zum kognitiven und voluntativen Grund der Gotteserfahrung nach Bonaventura (Münchener Universitätsschriften. Veröffentlichungen des Grabmann-Institutes, NF. 35), Paderborn 1990, S. 149, Anm. 86. 
einem der wichtigsten Autoren zum christlichen Liebesbegriff - dem Tastsinn zugeordnet ${ }^{43}$. In dieser Weise interpretiert bedeutet der Handschlag nicht nur im juridischen Sinne das Abschließen eines Vertrages, sondern belegt zugleich den Akt der Verwandtenliebe, die hier mit den Händen erfühlt wird.

In der zweiten Illustration der Seite findet die bisher nur vermutete didaktisch-moralische Ausrichtung insoferne eine weitere Bestätigung, als die Lehrhaftigkeit der Szenen durch die vermittelnde Erzählerfigur deutlich betont wird ${ }^{44}$. An Willehalm selbst ist eine wichtige Veränderung zu beobachten, die bis zur vorletzten Szene auf der Versoseite (Abb. 4) anhält: Er trägt das Schwert entgürtet und hat damit - im Sinne der durch die Liebe seiner Mutter eingeleiteten Versöhnung - den im Zorn gegen die Schwester vorgenommenen Verstoß aufgehoben. Damit erst wird die Umstimmung, die Alyze durch ihre Lieblichkeit eingeleitet hat, Schritt für Schritt vollendet. Diese verschiedenen Stationen der wiederhergestellten Ordnung werden nun, etwa in der lehrhaften Art der Bergpredigt im sogenannten Gebetbuch der Hildegard von Bingen, hintereinander als die verschiedenen Formen christlicher Liebe exemplifiziert. Gerade in diesen Szenen sind Text und Bild nicht synchron angeordnet, sondern in den Illustrationen werden Vor- und Rückgriffe vorgenommen. Im Gegensatz zu Werner Schröder ${ }^{45}$, der dieses Vorgehen als Beweis für die verfehlten Mittel der Darstellung wertet, möchte ich darin jedoch ihre didaktische Vermittlungsabsicht sehen und behaupten, daß mit Hilfe dieser Bildmittel der Text dazu benutzt wird, die einzelnen Erscheinungsweisen der Caritas bzw. letztendlich der Liebe zu Gott zu erklären ${ }^{46}$.

In der zweiten Darstellung beginnt diejenige Sequenz, in der Willehalms Schwester selbst versucht, ihre Schuld zu sühnen. Hier wird im Vorgriff bereits eine Szene ins Bild übertragen, die im gegenüberstehenden Text lediglich angekündigt (VV. 162, 5-7) und erst an einer späteren Stelle des Epos geschildert wird (VV. 164, 10 ff.): Im gemeinsamen Schmerz und Trauer um die Gefallenen, durch das Mitleid, das Bruder und Schwester miteinander teilen und das - wie seine Hand auf dem Herzen bekräftigt - sie beide tief berührt, sind sie miteinander verbunden. Hierin - so wird der Betrachter belehrt - liegt der Weg zur Versöhnung. Die überkreuzten Arme des Erzählers, eine Geste, die in der Regel negative Assoziationen vermittelt und meist in Verratsszenen oder bei Meineid eingesetzt wird ${ }^{47}$, dürfte hier im Sinne einer emotionalen wie auch zeitlichen Ambivalenz zu verstehen sein. Sie macht darauf aufmerksam, daß zu diesem Zeitpunkt die Konfliktsituation noch nicht abgeschlossen ist, und weist zugleich auf die kommende Veränderung hin ${ }^{48}$. Damit ist vorauseilend bereits in dieser Darstellung auf die nachfolgenden Geschehnisse verwiesen, in denen erst nämlich in der zweiten Szene der Versoseite - die Versöhnung erreicht und damit die Ambivalenz beendet sein wird. In dieser zweiten Szene ist nun offensichtlich, daß der

43) K. RuH, Die Augen der Liebe bei Wilhelm von St. Thierry, in: Theologische Zeitschrift, 45, 1989 (Festschrift für Martin Anton Schmidt zum 70. Geburtstag), S. 103-114, bes. S. 104.

44) Dazu OTr, Körper (xit. Anm. 26), S. 224.

45) SCHRODER, Text und Bild (zit. Anm. 17), S. 247.

46) Curschmann, Pictura (zit. Anm. 26), S. 220, versteht die Illustrationstechnik ebenfalls als "Mißgriff“, betont aber zugleich, man habe hier das "Bild im ,Litteraten'-Sinn, lesbar' machen wollen".

47) F. GARNIER, Le langage de l'image au moyen âge. Signification et symbolique, Paris 1982, S. 216-222.

48) GarniER, ebenda, S. 222, weist auf eine Darstellung in Le Mans hin, in der Jakob in dieser Weise seinen Segen an Ephraim und Joseph erteilt. 
Gegenstand der Bilder die christliche Liebeslehre ist. Hier wird einer der wichtigsten Grundsätze dieser Lehre thematisiert, der dann in den nachfolgenden Bildern in verschiedenen Versionen näher ausgeführt wird, nämlich der amor deficiens. Nicht um Verwandtenliebe geht es hier, sondern das Mitleiden mit den Todgeweihten ist Gegenstand dieser Darstellung, die damit im Vergleich zur ersten Szene eine höhere Form von Caritas verbildlicht. Die Verwandtenliebe, im ersten Bild festgehalten und hier ebenfalls die erste Regung, hat, da den betrauerten Angehörigen der Tod bei der bevorstehenden Schlacht gewiß ist, den Charakter des amor deficiens, einer vergeblichen Liebe, erreicht. Diese nun umfaßt gemäß Wilhelm von St.-Thierry keineswegs nur negative Aspekte, sondern stellt den Liebenden im Gegenteil in die Nachfolge Christi, muß er doch entsprechend "Hinfälligkeit und Gnadenlosigkeit" ertragen 49 . Damir aber wird die vergebliche Liebe zugleich auch "Garant von Auferstehung und Heilserwartung".

Der amor deficiens ist in der nächsten Szene ebenfalls Thema der Darstellung, in der eine andere Variante dieser Liebe exemplifiziert wird: die Angst Willehalms um seine in Orange von den Heiden belagerte Gattin Giburg. Diese Sorge ist ja auch, wie wir uns erinnern, Anlaß für den unbändigen Zorn gegen seine Schwester. In einer Trauerhaltung, mit der an die Wange gelegten, stützenden Hand, wie wir sie etwa in den ${ }_{\text {Beati qui lugent }}{ }^{\text {des }}$ Wiener Exemplars des sogenannten Gebetbuchs der Hildegard von Bingen kennen (Abb. 2), fürchtet Willehalm um seine Gattin. Wenn auch das Schicksal der Verwandten schmerzt, wie viel leidvoller ist noch die Not um Giburg (VV. 162, 25 f.): mitten in seinem herzen lac / gruntveste der sorgen fundamint. Giburg was sin liebistez pfant / nach ir im sine sinne und freude swant (VV. 162, 19 f.). Nach dem Ideal der höfischen Minne wäre der verzehrende Schmerz und das Außersichgeraten Willehalms allein schon durch die Trennung der beiden Partner sanktioniert ${ }^{50}$. Hier jedoch wird sozusagen eine sublimierte Version dieses Liebesleides gezeigt. Nicht allein die Sehnsucht nach der Geliebten ist Grund für Willehalms Trauerhaltung, sondern, und darauf weist die Gestalt des Erzählers mit belehrender Geste hin, ihre Lage, das Eingesperrtsein in Orange löst in Willehalm diesen Schmerz des Mitleidens, der Compassio aus. Mit den „Augen des Herzens" (oculi cordis) wird Willehalm ihrer in der Ferne in einer Burg ansichtig. Daß damit nicht das Thema der unerreichbaren Liebe in der Minneburg angesprochen wird, sondern ihr Eingesperrtsein als schicksalhaftes Geschehen interpretiert sein soll, wird durch den neben der Burg schwebenden Wiurfel verdeutlicht. Der darauf sichtbare, im Text genannte eine Punkt ${ }^{51}$ bezeugt nicht nur das von niemandem, auch nicht mit einem einzigen Punkt mehr zu übertreffende Ausmaß seiner Unrast, sondern belegt auch in diesem Bild des Würfelspiels Willehalms Hingabe an das Schicksal, für welches die Metapher des Würfelspiels steht ${ }^{52}$. Willehalms

49) K. RuH, Geistliche Liebeslehren des XII. Jahrhunderts, in: Beiträge zur Geschichte der deutschen Sprache und Literatur, 111, 1989, S. 157-178, bes. S. 164. Die Hand, die Willehalm auf sein Herz gelegt har, charakterisiert die Reinheit des Herzens; dazu s. Schmrrt, Gestes (zit. Anm. 32), Abb. 16.

50) Zum Begriff der höfischen Minne auch in der Ehe vgl. die Übersicht bei R. ScHNell, Causa amoris. Liebeskonzeption und Liebesdarstellung in der mittelalterlichen Literatur, Bern 1985, S. 115 ff., bes. S. 125; zum Verständnis der verzehrenden Liebe als Liebeskrankheit, die ebenfalls einen Normenbruch erlaubt, ebenda, S. 244 ff.; zum Minnebegriff im „Willehalm" s. KuENing, Reflexion-Narration (zit. Anm. 39), S. 169 ff.

51) Willehalm (zit. Anm. 1), V. 162, 22.

52) Dazu J. Hennzle (Hrsg.), Wolfram von Eschenbachs "Willehalm“. Bibliothek des Mittelalters, hrsg. von Walter Haug, 9, Frankfurt am Main 1991, S. 951; vgl. auch OrT, Körper (zit. Anm. 26), S. 225 und Anm. 6. 
Liebesleid wird damit zu einer der höchsten Stufen christlicher Liebe, zu einem amor deficiens, in dem das Leiden in der Nachfolge Christi verstanden wird.

Die wohl erstaunlichste Darstellung in diesem Lobpreis der Caritas findet sich im oberen Register der Versoseite (Abb. 4). Hier wird gezeigt, wie sogar die Juden und Heiden von der Tiefe des Leidens, das über Willehalm gekommen ist, berührt werden. Was im Epos nur im Konjunktiv vorstellbar ist: er möht erbarmen die halt sint, / des waren gelouben ane, / juden, beiden und publicane 53 , wird hier durch die gemeinsam mit Willehalm travernden Juden und Heiden verbildlicht. Während der Heide seine Wange in die Hand legt und damit weniger ausdrucksstark den Willehalmschen Trauergestus nachvollzieht ${ }^{54}$, legt der vor diesem stehende Jude seine Hand an den Mund. Wiederum erschließr sich der Sinn dieser Gesten aus der Lehre des Wilhelm von St.-Thierry. Die hier dargestellte Form der Caritas, die Feindesliebe, gehört zur sozialen Liebe. Diese ist, wie die Geste des Juden bezeugt, dem Geschmackssinn zugeordnet ${ }^{55}$. Darüber hinaus allerdings ist in dieser Szene ein weiterer wichtiger Grundsatz der Caritastheorie angesprochen, kommt doch nach diesen monastischen Lehren jede Liebe von Gott und erfaßt somit - hier im Bild bekräftigt - auch den Andersgläubigen ${ }^{56}$.

In der mittleren Szene wird das Wunder der Liebe mit all seinen Folgen beschrieben. Willehalm, nun wieder mit gegürtetem Schwert und das Bild der Geliebten als Vision vor Augen ${ }^{57}$, trauert trotz seiner demonstrativen Kampfbereitschaft um die voraussichtlichen Opfer. Was in der vorangehenden Seite als bevorstehendes Ereignis vorweggenommen worden war, hat sich hier erfüllt. Auch Willehalms Schwester steht nun, immer noch ohne die Krone, die ihr ja der Bruder vom Kopf gerissen hatte, in tiefer Trauer neben den Toten. Die Geschwister sind somit hier in derselben Haltung vereint, während in der dieses Ereignis ankündigenden Szene auf der Rectoseite des Blattes die Schwester lediglich mit einem Sprechgestus ihre Betroffenheit zeigt. In der diese Sequenz beendenden Darstellung schließlich wird die Königin gezeigt, wie sie aus Angst vor der zerstörerischen Wut ihres Bruders versucht, Alyze am Eindringen in ihr Gemach zu hindern. Das Eingesperrtsein wird hier zur Metapher des ${ }_{n}$ verschlossenen Herzens ${ }^{\text {", }}$, das durch die gemeinsame Liebe von Schwester und Bruder im Sinne des vorher exemplifizierten christlichen Liebesbegriffs geöffnet wird ${ }^{58}$.

Fassen wir die auf diesem Blatt gezeigten Inhalte zusammen, so bildet in allen Darstellungen die Kraft der Liebe den thematischen Mittelpunkt. Die anrührende Schilderung der Gefühlslagen des Helden ist allein in diesen Zusammenhang gestellt. Nicht die affektgeladene Stimmung Willehalms, sein Zorn oder seine Liebesentbehrung werden thematisiert, sondern es werden Emotionen gezeigt, die in den Kontext christlicher Liebeslehren gehören. Es wird denn auch niemals Liebe im Sinne des Minneideals angesprochen, wie man es

53) Willehalm (zit. Anm. 1), VV. 162, 28-30; dazu HeinzLE, ebenda, S. 952.

54) $\mathrm{Zu}$ den Unterschieden von Verzweiflung, Trauer und Melancholie in den Gesten vgl. Barash, Gestures (zit. Anm. 7), S. 36 ff.; vgl. auch die Differenzen in den sogenannten Versionen der Gebetbücher Hildegards von Bingen, dazu ScHMrT, Gestes (zit. Anm. 32), Abb. 17,2.

55) Rur, Liebeslehren (zit. Anm. 49), S. 168.

56) Zur Feindesliebe etwa bei Bernhard von Clairvaux vgl. RuH, ebenda, S. 168.

57) Zur Bedeutung der Vision als Erleuchtung vgl. RuH, Augen (zit. Anm. 43), S. $105 \mathrm{f}$.

58) Zur Metapher des Herzens als Wohnung vgl. F. OHLY, Cor amantis non angustum. Vom Wohnen im Herzen, in: DeRs., Schriften zur mittelalterlichen Bedeutungsforschung, Darmstadt 1977, S. 128-155, bes. S. 129 f. (zuerst 1970). 
bei einem höfischen Epos eigentlich erwarten würde, sondern in ungewöhnlicher Präzision werden die einzelnen Formen der Caritas dargelegt: die Liebe zu den Verwandten, zum Nächsten, aber auch zum Feind wird hier zum Vorbild erhoben. Besonderen Raum allerdings erhält die leidende Liebe im Sinne der Hingabe an das Unausweichliche, an die Passion, die mit Christus verbindet und damit zugleich Erlösung verspricht.

Wenn man diese Illustrationen als Lehrbilder zum Vorstellungskreis der Caritas versteht, dann wird ersichtlich, daß sie nicht so sehr dazu dienen, das Epos zu verbildlichen, als im Gegenteil dieses dazu benützt wird, andere Inhalte zu übermitteln. Allerdings muß darauf hingewiesen werden, daß sie - wie insbesondere die Vergleiche zum Text gezeigt haben diesem keineswegs widersprechen, vielmehr gehen sie mit ihrer prononcierten Stellungnahme weit über ihn hinaus und isolieren dabei eine Thematik, die im Epos nur angelegt ist. Alle Elemente dieser Liebeslehre in "Lesebildern" sind derart klar herausgearbeitet und in einzelne Lehrschritte untergliedert, daß die Sequenz nicht allein der Illustration einer solchen Lehre dienen kann. An ihnen sollen nicht nur die Formen christlicher Liebe exemplifiziert werden, sondern es gilt, diese - wofür die ähnlich strukturierte Bebilderung des sogenannten Gebetbuchs der Hildegard von Bingen eine Parallele zu bieten scheint - in der „Bildlesung" nachzuerleben. Willehalms Kraft als Vorbild, dank der er sowohl Schwester als auch Juden und Heiden dazu bewegte, über den affektiven Zugang sein Lieben und Leiden zu teilen, soll auch auf den Betrachter wirken. Es ist das Mitleiden, was auch ihm helfen wird, der Erlösung teilhaftig zu werden.

Die Theorien, in denen dieser Zusammenhang von Liebe, Leiden und Erlösung artikuliert wurden, weisen auf einen monastischen Hintergrund hin. Weniger zeitgenössische Lehren als vielmehr die Literatur des 12. Jahrhunderts, insbesondere Anschauungen des Wilhelm von St.-Thierry scheinen dabei berücksichtigt worden zu sein. Um die theologische Auffassung der Bilder und insbesondere die in sie eingefloßenen monastischen Caritastheorien zu verdeutlichen, wollen wir uns den Illustrationen zum sogenannten Religionsgespräch zwischen Giburg und Teramer ${ }^{59}$ zuwenden. Hier wird nämlich eines der damals brisantesten theologischen Themen abgehandelt: die Konzeption der Dreieinigkeit ${ }^{60}$.

$\mathrm{Zu}$ Beginn des Religionsgesprächs, das die in Orange gefangene Giburg mit ihrem sie belagernden Vater Teramer führt, preist sie zunächst die Wunder der Schöpfung und deren Schöpfer (Abb. 5). Dieser Lobpreis ist in den beiden oberen Registern des Nürnberger Fragments dargestellt. Darunter trauert Teramer über den Abfall seiner Tochter vom „richtigen" Glauben. Auf der Versoseite dieses Blattes dann (Abb. 6) erklärt der auf seinem Pferd in voller Rüstung sitzende Teramer seiner Tochter Giburg, er habe seine Verpflichtung gegenüber den heidnischen Göttern - diese sind in der üblichen Form des nackten Idols am Bildrand sichtbar - über seine Vaterpflicht gestellt. Eindringlich versucht Giburg daraufhin ihren Vater von der Notwendigkeit der Erlösung zu überzeugen, da auf der Menschheit die

59) Nürnberg, Germanisches Nationalmuseum: Inv. Nr. Hz 1104 und 1105.

60) Zur Analyse der Bilder vgl. SCHrODER, Text und Bild (zit. Anm. 17), S. 249 f. - Uber die engen Beziehungen des Textes zu theologischem Schriftum vgl. etwa F. OHLY, Wolframs Gebet an den Heiligen Geist im Eingang des "Willehalm", in: Wolfram von Eschenbach, hrsg. von H. Rupp (Wege der Forschung, 57), Darmstadt 1966 (zuerst 1961/62), S. 455-518, bes. S. 506; A. KrelpINSKI, Der Heide Rennewart als Heilswerkzeug Gottes. Die laientheologischen Implikationen im "Willehalm" Wolframs von Eschenbach, phil. Diss., Berlin 1990, bes. S. 147, belegt für den Text einen allgemeinen christianisierenden Charakter im Vergleich zur monastischen Ausrichtung der Illustrationen. 
Erbsünde laste. Dem Text entsprechend wird im zweiren Register (VV. 218 ff.) die Erbsünde nicht durch den Sündenfall verbildlicht - womit Bild und Text gleichermaßen der zeitgenössischen Theologie folgen -, sondern als die eigentliche Urheberin wird hier Eva dargestellt. Gemäß damaliger Interpretation erlag Eva, infolge mangelnder Einsicht, den Einflüsterungen Satans, während Adam lediglich Mitschuldiger war ${ }^{61}$. Im dritten Register schließlich beschwört Giburg die Konsequenz der Verdammnis (VV. 218, 16 ff.), die wir in einem Teufel verbildlicht sehen, welcher eines der nackten Kinder Adams in den Höllenrachen schiebt. Darüber jedoch ragt als Garant der Erlösung das Bild der Trinität (V. 218, 25).

In dieser letzten Darstellung, insbesondere in der Wiedergabe der Dreieinigkeit, kommen nun Gedanken zum Tragen, die uns ermöglichen, das bisher eruierte theologische Umfeld etwas genauer zu konkretisieren. In einem Kreis, dem Trinitätssymbol an sich ${ }^{62}$, sind drei gleichartige Köpfe zu sehen, wobei der obere, der die beiden anderen überragt und von einem Kreuznimbus überstrahlt ist, eindeutig als höchstes Prinzip ausgezeichnet ist. Von diesem leiten sich die zwei anderen Personen zwar ab, zugleich aber wird bei allen ihre Wesensgleich heit deutlich gemacht.

In diesem einfachen Konzept einer Trinitätsdarstellung spiegelt sich die zeitgenössische Diskussion des „Filioque ${ }^{“}$. Hierzu wird im Bild folgendermaßen Stellung genommen: Durch den Kreuznimbus wird die enge Verbindung zwischen Vater und Sohn betont, ist jener damit doch selbst als Urgrund der Menschwerdung und allen Leidens Christi bezeichnet. Trotz der prinzipiellen Wesensgleichheit aller drei Personen wird dennoch kein Zweifel an der Überordnung der ersten über die beiden anderen gelassen. Ein solches Verständnis der Dreieinigkeit entspricht der Trinitätslehre, wie sie schon von den Viktorinern und insbesondere in deren Rezeption von der franziskanischen Theologie vertreten wurde. Vor allem in Bonaventuras Trinitätsdeutung finden sich die entsprechenden Aussagen: ${ }_{n}$ Als der Menschgewordene, Gekreuzigte und Auferstandene ist Christus die Offenbarung des dreifaltigen Gottes. In Christus ist der Erste verbunden mit dem Letzten ${ }^{463}$. In seinen Schriften betont Bonaventura auch die absolute Wesensgleichheit der drei Personen und deren entsprechende Gleichförmigkeit64: Der Heilige Geist ist "Gabe, Band oder Wechselliebe ... "65; er ist Liebesgabe Gottes, der die Erlösung vollendet ${ }^{66}$.

Was die Caritasbilder noch nicht an Einordnungsmöglichkeiten des ihnen zugrundeliegenden Gedankenguts liefern konnten, vermögen infolgedessen diese Illustrationen näher zu konkretisieren. Nicht bloß ein allgemein monastischer Hintergrund scheint in ihnen eine Rolle zu spielen, sondern gerade die hier verbildlichten Konzepte der Trinität und der

61) Vgl. dazu H. KOSTER, Urstand, Fall und Erbsünde in der Scholastik, in: Handbuch der Dogmengeschichte, hrsg. von M. Schmus u. a., Bd. 2: Der Trinitarische Gott. Die Schöpfung und die Sünde, Freiburg i. B. 1979, bes. S. 110-114.

62) Vgl. dazu A. EgGer, Das höchste Kunstproblem: Die Darstellung der Dreieinigkeit, in: Der Schlern, 24, 1950, S. 100-107; Dreifaltigkeit, in: Reallexikon zur Deutschen Kunstgeschichte, hrsg. von E. GAul u. a., Bd. 4, Stuttgart 1958, Sp. 414 ff.; D. DENNY, The Trinity in Enguerrand Quarton's Coronation of the Virgin, in: The Art Bulletin, 1963, S. 48-52.

63) F. CourTh, Trinität in der Scholastik, in: Schmaus, Handbuch, Bd. 2 (zit. Anm. 61), S. 134.

64) Ebenda, S. 132.

65) Ebenda, S. 133.

\$) Ebenda, S. 134. 
Erbsünde weisen auf einen franziskanischen Zusammenhang hin. In diesen Rahmen fügen sich auch die Bilder zu Willehalms Versöhnung nahtlos ein. Geht es doch gerade dabei wie auch im Trinitätsverständnis Bonaventuras - im wesentlichen darum, ein Konzept der christlichen Liebe zu zeigen, in der diese als Liebesgabe Gottes verstanden wird, dank der nicht nur Feinde geliebt werden können, sondern sogar über den amor deficiens die Erlösung garantiert wird.

Kehren wir zu unserer Eingangsthese zurück, es bestehe eine Scheu vor der Verbildlichung von Affektzuständen. Die zunächst unerwartet intensive Darstellung von Gemütszuständen, in denen Willehalm gezeigt wird, hat diese Behauptung weiter bestätigt. Thematisiert werden hier nicht seine subjektiven Gefühle von Zorn oder die Verlerzung einer Norm, sondern der Ausbruch des Helden wird im Gegenteil dazu verwendet, die Vorbildlichkeit bestimmter Gefühle zu exemplifizieren. Im Bild ist somit die Emotionalität des literarischen Epos in eine andere Gefühlslage uminterpretiert worden, ja das gesamte Werk hat durch die Bilder eine neue Richtung erhalten. In diesen beiden Blättern wird die Wahl einer Illustrationsweise, welche ihre nächste Parallele weniger in den Sachsenspiegeln als von ihrer Vermittlungsabsicht her eher in den theologisch-didaktischen Werken in der Art der sogenannten Hildegard-Gebetbücher hat, als programmatisch verständlich und läßt nichts von einem Mißgriff erkennen. Wie weit man nun aus den wenigen Seiten, die allerdings innerhalb der erhaltenen Bruchstücke besonders aussagekräftig zu sein scheinen, auch weitergehende Schlüsse über die Vermittlungsabsicht und die möglichen Rezipienten des ursprünglichen Kodex ziehen kann, muß hier unerörtert bleiben. Was jedoch die Frage nach der Darstellbarkeit von Emotionalität angehr, so wird in der Sequenz über die verschiedenen Formen der Caritas sowohl für den Helden als auch für den Betrachter eben jener Teil affektiven Erlebens verbildlicht, der beiden nicht bloß erlaubt, sondern geradezu zur Nachahmung empfohlen wird.

Abbildungsnachweis: Abb. 1, 2: Wien, Osterreichische Nationalbibliothek. - Abb. 3, 4: München, Bayerische Staatsbibliothek. - Abb. 5, 6: Nürnberg, Germanisches Nationalmuseum. 

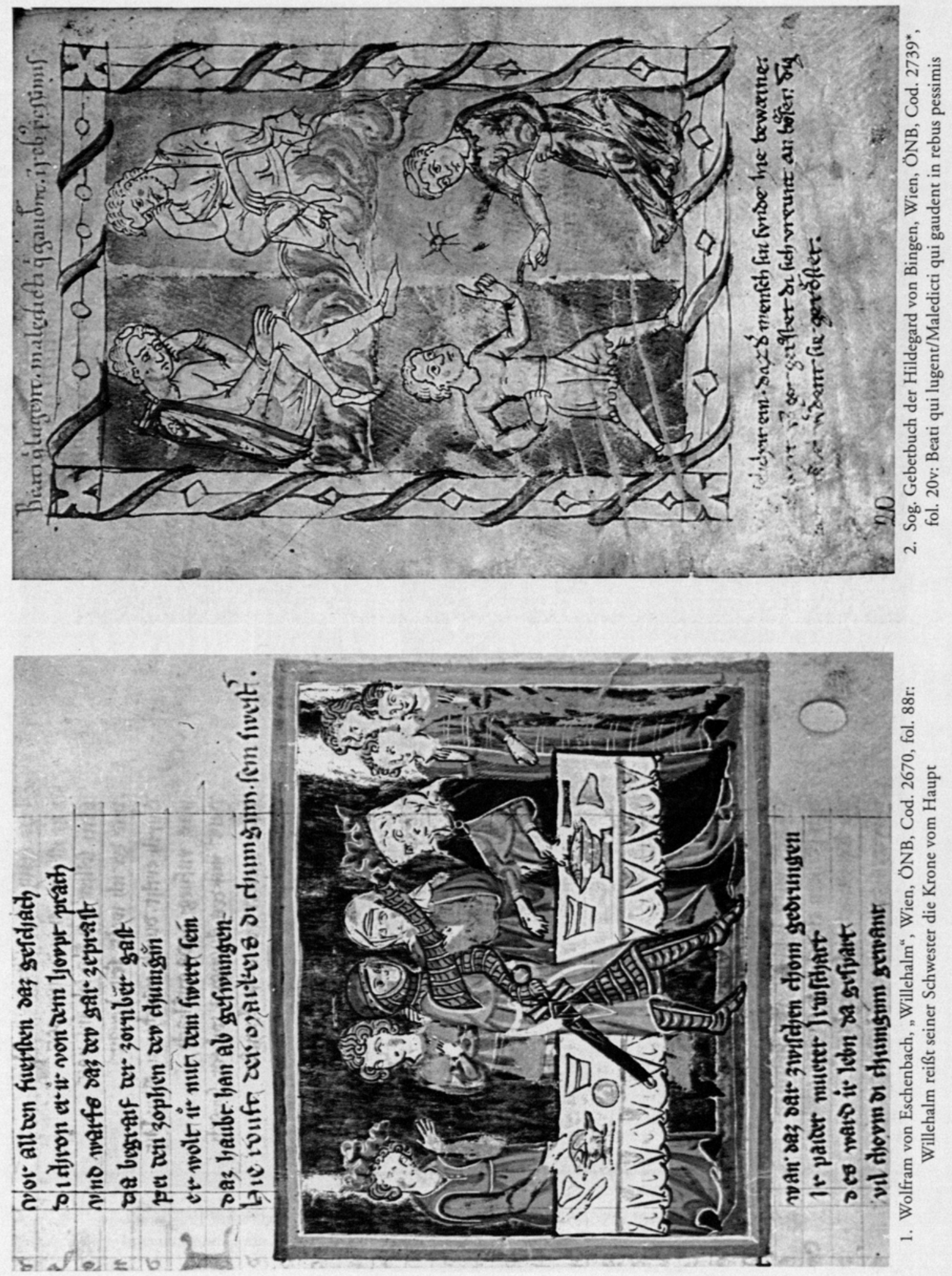


\section{$\operatorname{Cgm} 193, \pi 1$, 1 (45)}

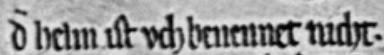
Dociano mufien noth o fbult.

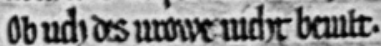
soder mir firs unuer thine.

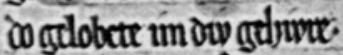
bonfilb ron golde ton anocen folic: tos menoer to out grenod).

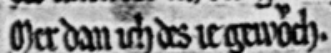

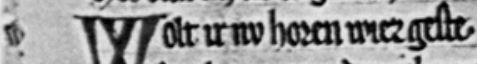

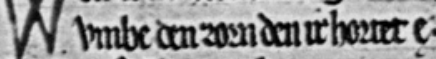

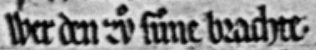
the dem markife nadjec:

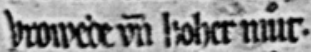

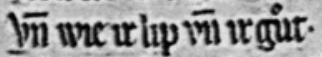

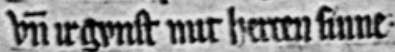
otw romuldo kntumgunc. g) re muwengap in fin grbot. as uns kisbume not. co ocmmatkif wolgelame. den mume ที 1 amer tutunc. line pfanios her listaner west. Alv prubet ouditen gwosen mayt. Jufaliyfamzadcadj.

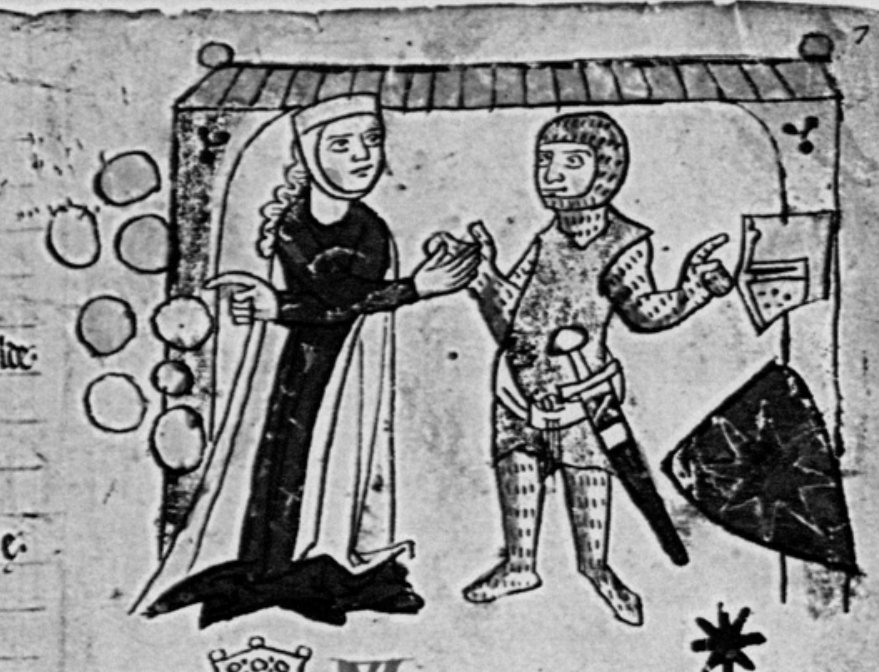

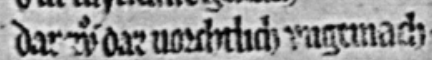
dakylutur tumeblep.

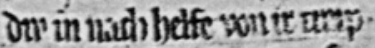
kyisure uni fin lidofte yin: Aadis tm tum urome fwuss.

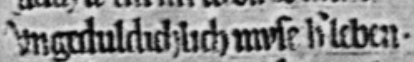

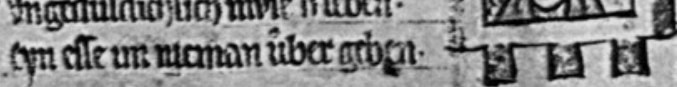
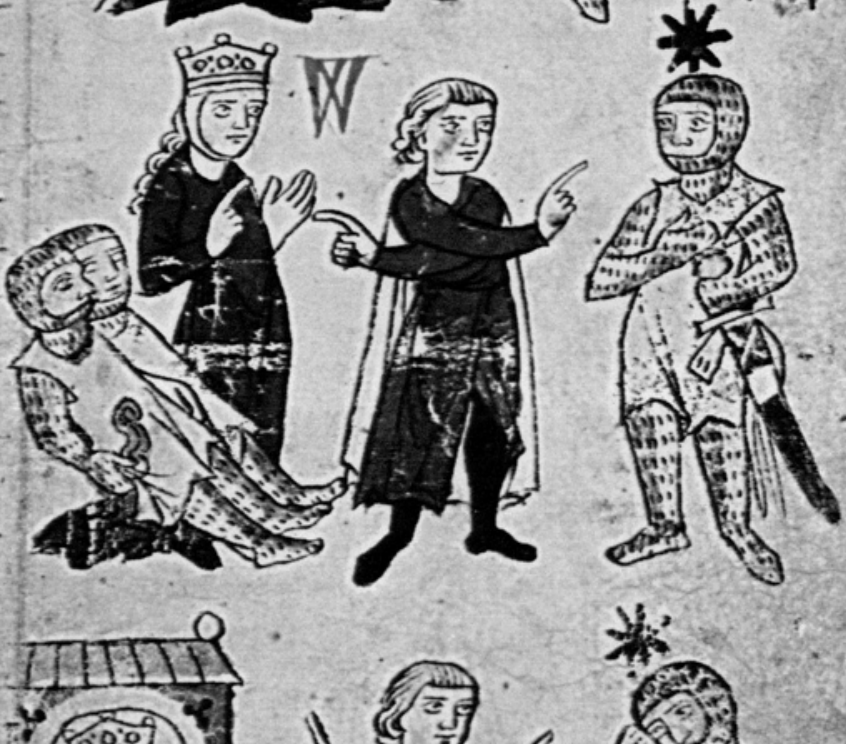

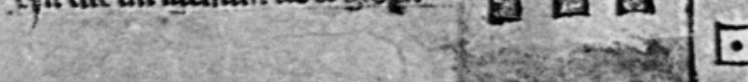
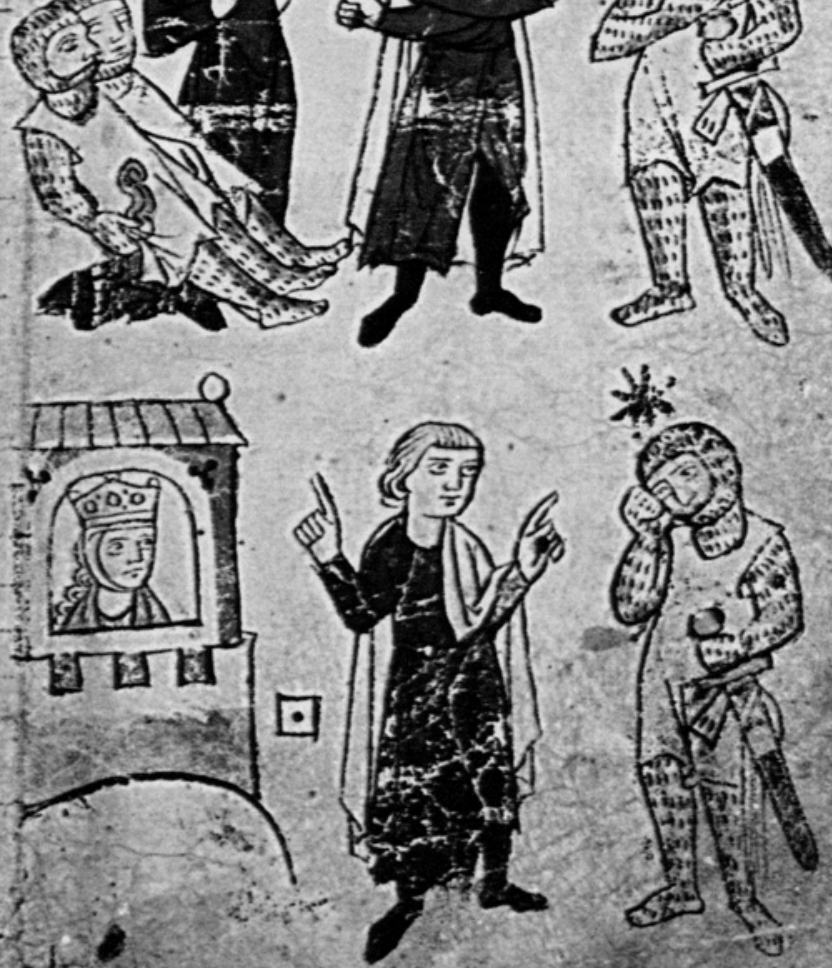

3. Wolfram von Eschenbach, „Willehalm“, München, Bayer. Staatsbibliothek, Cgm 193/III, fol. 1r: Willehalm und Irminschart, Willehalm mit seiner Schwester, Willehalm um Giburg trauernd 


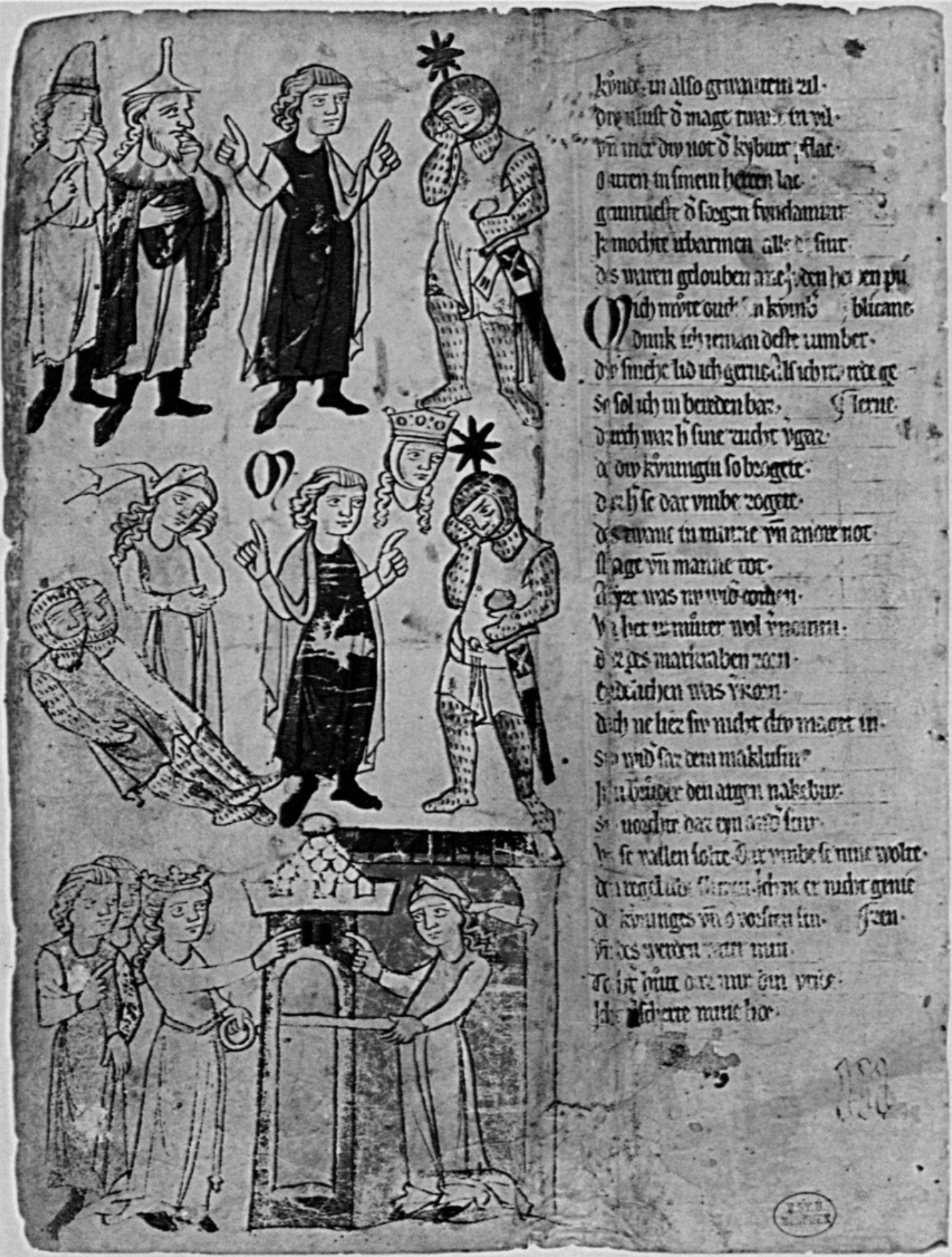

4. Wie Abb. 3, fol. 1v: Willehalm mit Heiden und Juden, Willehalm mit seiner Schwester, die Schwester flüchtet vor Willehalms Zorn 

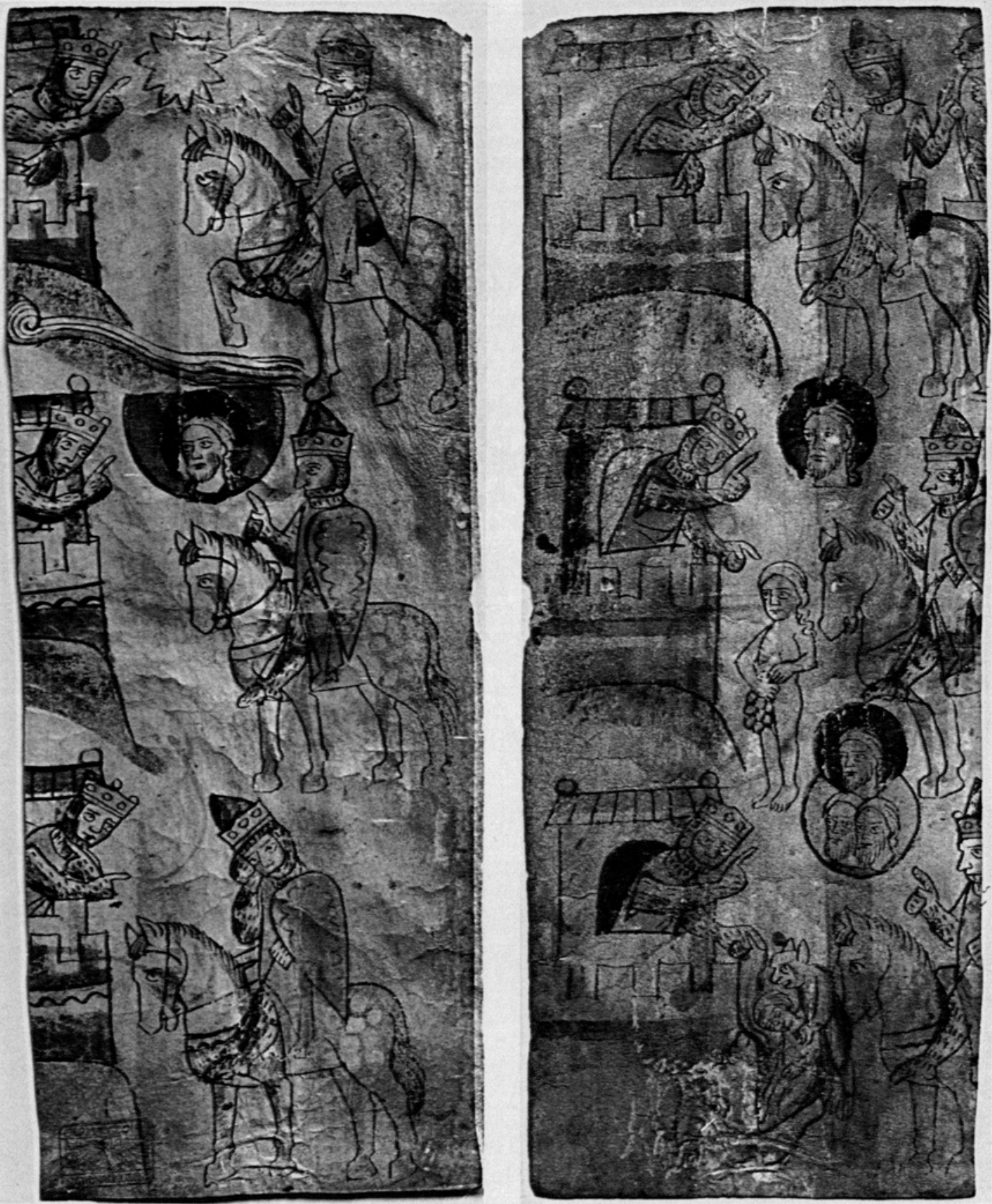

5, 6. Wolfram von Eschenbach, „Willehalm“, Nürnberg, Germanisches Nationalmuseum, Kupferstichkab., $\mathrm{Hz} 1104$ und 1105: Religionsgespräch zwischen Giburg und Teramer 